\title{
Degradation of p53 by natural variants of the E6 protein of human papillomavirus type 16
}

\author{
JA WOON YI* , MI JANG* ${ }^{*}$ SUNG JIN KIM, SUNG SOON KIM and JEE EUN RHEE \\ Division of AIDS, Center for Immunology and Pathology, Korea National Institute of Health, Seoul, Republic of Korea
}

Received October 5, 2012; Accepted December 17, 2012

DOI: $10.3892 /$ or.2013.2281

\begin{abstract}
The degradation of p53 by high-risk human papillomavirus (HR-HPV) E6 proteins is recognized as necessary for the immortalization of mammary epithelial cells and the progression of cancer. The HR-HPV type 16 E6 proteins exhibit numerous variants associated with different risk factors for the development of cervical cancer. Two variants of E6 proteins, D25E and L83V, are common in cervical carcinomas among Asian and European populations. In the present study, we compared the effect of two E6 variants on p53 degradation by a prototype E6 protein. We demonstrate that both the D25E and L83V variants downregulate p53 through a ubiquitinproteasome pathway, and that the effect is very similar to that of the prototype E6 protein. The reduction in the p53 protein levels was induced through the ubiquitin-proteasome pathway via interaction with E6 proteins. The expression of $\mathrm{p} 21$ CIP1/WAF1, a downstream molecule of p53, was similarly reduced in both prototype and variant E6 protein-expressing cell lines, leading to aberrant G1/S cell cycle arrest. These results suggest that the natural variants, E6 D25E and L83V, similar to the prototype E6 protein, contribute to tumorigenesis by degrading p53.
\end{abstract}

\section{Introduction}

The development of cervical carcinomas closely correlates with the presence of certain high-risk human papillomavirus (HR-HPV) types, such as HPV-16 and HPV-18. HPV-16 is the most common HPV type detected in cervical cancer, accounting for $50 \%$ of cancers and high-grade squamous intraepithelial lesions (1). Carcinogenesis relies primarily on the expression of two virally encoded oncoproteins, E6 and E7. These act synergistically to immortalize and transform the infected cells partly through their ability to degrade p53 and Rb, respectively (2-4).

Correspondence to: Dr Jee Eun Rhee, Division of AIDS, Center for Immunology and Pathology, Korea National Institute of Health, Seoul, Republic of Korea

E-mail: jeeeunrhee@gmail.com

*Contributed equally

Key words: human papillomavirus 16, E6 prototype, E6 variant, p53, p21, cell cycle
The HR-HPV E6 protein has been demonstrated to lead to the ubiquitin-mediated degradation of $\mathrm{p} 53$ by direct interaction with the cellular E3 ubiquitin ligase, E6AP (5). The specific action of E6 on p53 is functionally equivalent to p53 inactivation through mutation, which indicates that the HR-HPV E6/p53 complex represents one of the most important events in cervical carcinogenesis, given the interruption of the cell cycle control points and inhibition of apoptosis. The degradation of p53 induced by E6-AP is a significant effect of HR-HPV and results in the malignant transformation of cervical epithelial cells together with the inactivation of p53.

Genetic studies have shown natural amino acid variants within the HPV-16 E6 oncoprotein (6). Variation within the E6 gene leading to such changes in amino acids can alter the biological and immunogenic properties of the encoded proteins $(7,8)$. Several studies have shown the existence of a link between E6 variants and the elevated risk of cervical intraepithelial neoplasia and invasive cervical cancer $(9,10)$.

However, little is known about the consequence of sequence variants with respect to the function of E6. A previous study analyzed a few of the HPV-16 E6 variants and showed that amino acid changes can alter their ability to abrogate serum/ calcium-dependent differentiation leading to p53 degradation in vitro (11). Other studies have reported that the European variant, $\mathrm{L} 83 \mathrm{~V}$, is associated with an increased risk of developing invasive cervical carcinoma in the Swedish population (10), and enhances mitogen-activated protein kinase (MAPK) signaling and cooperative transformation with deregulated Notch1 signaling (12). Previous studies by us, as well as others have indicated that E6 D25E, the most prevalent variant type in Asian populations including Chinese (13), Japanese (14) and Korean populations (15), may have a unique oncogenic role through different genes assoicated with the regulation of apoptosis or the cell cycle, such as AIFM2 and RPL23 (16). In the present study, we performed a functional analysis of naturally occurring E6 variants (D25E and L83V) to investigate the role of E6 polymorphisms in the development of cervical cancer. The E6 variants were evaluated for their ability to induce p53 degradation and inhibit p53 transactivation by comparing them with the reference HPV-16 E6 protein.

\section{Materials and methods}

Cell culture. The human cervical carcinoma cell lines, C33A, $\mathrm{SiHa}$ and HeLa, were obtained from the American Type 
Culture Collection (Manassas, VA, USA). The C33A and HeLa cells were cultured in MEM Alpha medium (Gibco; Life Technologies, Carlsbad, CA, USA). The SiHa cells were cultured in RPMI-1640 medium (Gibco) supplemented with $10 \%$ fetal bovine serum (Gibco) and antibiotics $(100 \mathrm{U} / \mathrm{ml}$ of penicillin and $100 \mu \mathrm{g} / \mathrm{ml}$ of streptomycin), at $37^{\circ} \mathrm{C}$ under humidified $5 \% \mathrm{CO}_{2}$ in air.

Gene construction of expression vectors and transfection. Prototype E6 was cloned into the eukaryotic expression vector, pFN21A HaloTag ${ }^{\circledR}$ CMV Flexi ${ }^{\circledR}$ Vector (Promega, Madison, WI, USA) containing an N-terminal HaloTag as described in the manufacturer's instructions. E6 was amplified by polymerase chain reaction $(\mathrm{PCR})$ with primers including the restriction site for $S g f$ I or PmeI (underlined): 5'-CGAAGCGATCGCC ATGCACCAAAAGAGAACTGC-3' and 5'-CATCGTTT AAACTTACAGCGGGTTTCTCTAC-3' from a previously constructed cell line [Jang et al (16)]. The E6 D25E and L83V variants were acquired in the E6 prototype construct using the QuikChange ${ }^{\circledR}$ site-directed mutagenesis kit (Stratagene, La Jolla, CA, USA), according to the manufacturer's instructions. The primers used for the E6 D25E and L83V variants were as follows. For E6 D25E, the primers were 5'-ACAACTATACAT GAGATAATATTAG-3' and 5'-CTAATATTATCTCATGTA TAGTTGTTTG-3'; for E6 L83V, the primers were 5'-GACATTATTGTTATAGTGTGTATGGAACAACATT AG-3' and 5'-GTAATGTTGTTCCATACACACTATAA CAATAATGTC-3'. All vectors were analyzed by nucleotide sequencing. Confirmed clones were transfected into each cell line using FuGene X-treme GENE HP DNA transfection reagent (Roche Applied Science, Pleasanton, CA, USA) according to the manufacturer's instructions.

Western blot analysis. The C33A, HeLa and SiHa cells were transfected with constructs containing the E6 prototype and variants. At $24 \mathrm{~h}$ after transfection, cell extracts were obtained by lysis in a RIPA cell lysis buffer $(150 \mathrm{mM} \mathrm{NaCl}, 1 \%$ TritonX-100, $1 \%$ deoxycholic acid sodium salt, $0.1 \%$ SDS, $50 \mathrm{mM}$ Tris- $\mathrm{HCl}$, pH 7.5 and 2 mM EDTA) supplemented with complete protease inhibitor tablets (Roche Applied Science). The extracts were then fractionated by $10 \%$ sodium dodecyl sulfate-polyacrylamide gel electrophoresis (SDS-PAGE) and transferred onto a polyvinylidene fluoride immunoblot membrane (Millipore, Billerica, MA, USA). The blot was incubated successively with the primary and the secondary antibodies, and the resulting signal was detected using enhanced chemiluminescence (Intron Biotechnology, Seoul, Korea). The antibodies used in this study were as follows: anti-p53 (DO-1; Santa Cruz Biotechnology Inc., Santa Cruz, CA, USA), anti-Halo (Promega), anti-p21 (Cell Signaling Technology, Inc., Beverly, MA, USA) and anti$\beta$-actin (Cell Signaling Technology, Inc.). $\beta$-actin was used as the loading control. To examine the effect of the proteasome inhibitor, MG132, E6 protein-expressing HeLa cells were incubated with $10 \mu \mathrm{M}$ MG132 for $2 \mathrm{~h}$ prior to western blot analysis.

Halo pull-down assay. The interaction between E6 variant proteins and p53 was identified using the HaloTag ${ }^{\circledR}$ Mammalian Pull-Down System according to the manufacturer's instructions (Promega). Briefly, approximately $1-1.2 \times 10^{7}$ cells were washed with phosphate-buffered saline (PBS) and lysed in
$300 \mu \mathrm{l}$ of mammalian lysis buffer (GenDEPOT, Barker, TX, USA), containing protease inhibitor (Roche Applied Science). Aliquots of $300 \mu \mathrm{l}$ of clear cell lysate were diluted with $700 \mu \mathrm{l}$ of $1 \mathrm{X}$ TBS (50 mM Tris, $\mathrm{pH} 7.4$ and $150 \mathrm{mM} \mathrm{NaCl}$ ). Diluted cell extracts were incubated with equilibrated HaloLink ${ }^{\mathrm{TM}}$ resin (Promega) at $4^{\circ} \mathrm{C}$ for $3 \mathrm{~h}$. The beads were washed three times with $1 \mathrm{ml}$ of Promega resin equilibration/wash buffer (with protease inhibitor) and washed resins were resuspended in $35 \mu \mathrm{l}$ of SDS sample buffer and boiled for $5 \mathrm{~min}$. The precipitated complexes were analyzed by western blot analysis using an anti-p53 antibody.

Quantitative real-time ( $R T$ ) PCR. cDNA was synthesized from $5 \mu \mathrm{g}$ of total RNA using an Omniscript RT kit (Qiagen, Hilden, Germany). We used $1 \mu \mathrm{l}$ cDNA for quantitive RT-PCR amplification using a SYBR Supermix kit (Bio-Rad Laboratories, Richmond, CA, USA). Samples were subjected to 45 cycles of $95^{\circ} \mathrm{C}$ for $20 \mathrm{sec}$ and $60^{\circ} \mathrm{C}$ for $1 \mathrm{~min}$. PCR efficiency was determined by running serial dilutions of template cDNA and melting curve data were collected to assure PCR specificity. Each cDNA sample was analyzed in triplicate and the corresponding non-RT mRNA sample was included as the negative control. A $\beta$-actin primer was included in every plate as the internal loading control. The following primers were used for quantitative RT-PCR of the p21 and $\beta$-actin genes: p21 forward, 5'-GCGGAACAAGGAGTCAGACA-3' and reverse, 5'-GGAAGGTGTTTGGGGTCAGA-3'; $\beta$-actin forward, 5'-ATCTGGCACCACACCTTCTA-3' and reverse, 5'-GGATAGCACAGCCTGGATAC-3'.

Cell cycle analysis using flow cytometry. Cell cycle analysis was performed by flow cytometry with propidium iodide (PI) staining. In brief, HeLa cells $\left(1.2 \times 10^{6}\right.$ cells $/ 10 \mathrm{~cm}^{2}$ dish $)$ were transfected with the empty vector, and with the Halo-E6, HaloD25E and Halo-L83V constructs. After $24 \mathrm{~h}$, the harvested cells were fixed in cold $75 \%$ ethanol at $4^{\circ} \mathrm{C}$ for $2 \mathrm{~h}$ and washed twice with PBS. The cells were stained with $0.5 \mathrm{ml}$ of $20 \mathrm{mg} / \mathrm{ml}$ PI containing $0.1 \mathrm{mg} / \mathrm{ml}$ RNase in PBS for $30 \mathrm{~min}$ at room temperature. DNA contents in 10,000 cells were analyzed with ModFit LT software (Verity Software House, Topsham, ME, USA) on a flow cytometer by gating on an area versus width dot plot to exclude cell debris and aggregates.

Statistical analysis. Data of activity in the various functional assays are presented in the figures as the means \pm standard deviation (SD). Data were subjected to a one-way ANOVA. A value of $\mathrm{P}<0.05$ was considered to indicate a statistically significant difference.

\section{Results}

E6 D25E and L83V variants reduce the levels of p53 expression in several cervical carcinoma cell lines. The targeting of p53 for degradation is believed to be an essential event in HPV-mediated malignant cell transformation (17). We investigated the ability of the E6 variants to degrade p53 in transient expression assays using Halo-tagged constructs expressing E6 proteins. The constructed DNAs were confirmed through sequencing analysis (data not shown). In our study, we used HPV-18-positive HeLa, HPV-16-positive SiHa and 

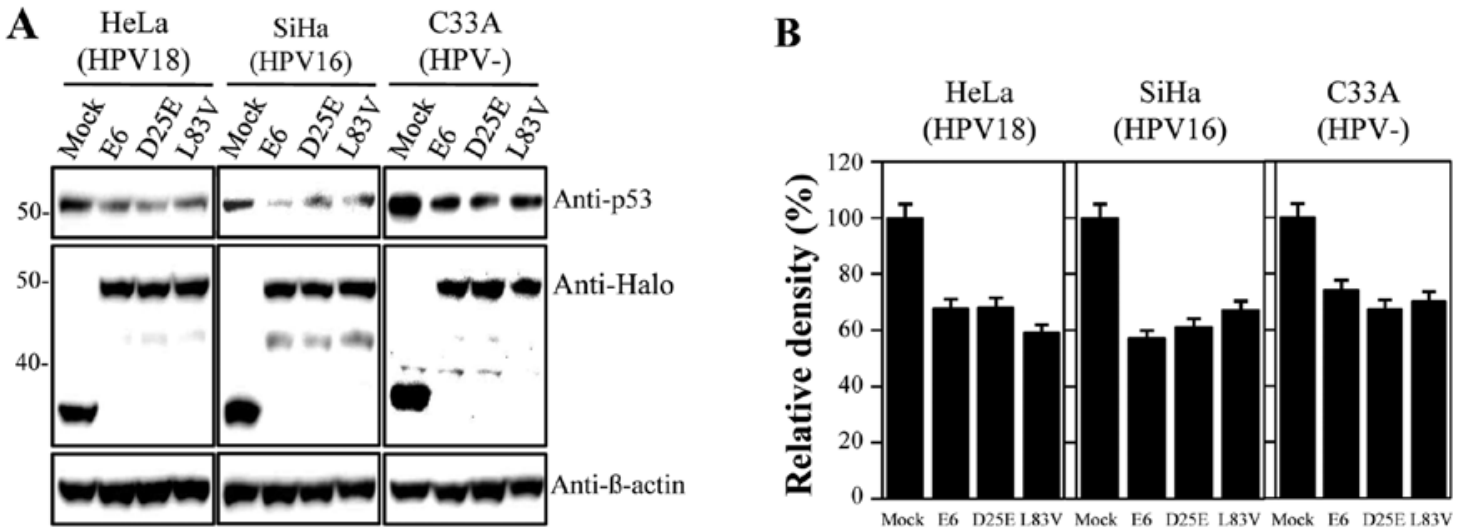

Figure 1. E6 D25E and L83V variants induce the degradation of p53. (A) HeLa, SiHa and control C33A HPV-negative cells were transfected with empty vector (Mock), Halo-E6, Halo-D25E and Halo-L83V vectors. Twenty-four hours after transfection, Halo-E6 and p53 expression levels were analyzed by western blot analysis using anti-p53 and anti-Halo antibodies. The result for $\beta$-actin is shown as the loading control in the bottom panel. (B) Relative densities of p53 proteins were determined from scanned western blots using ImageQuant and the signals in the cell lines transfected with the empty vector were defined as $100 \%$.

A

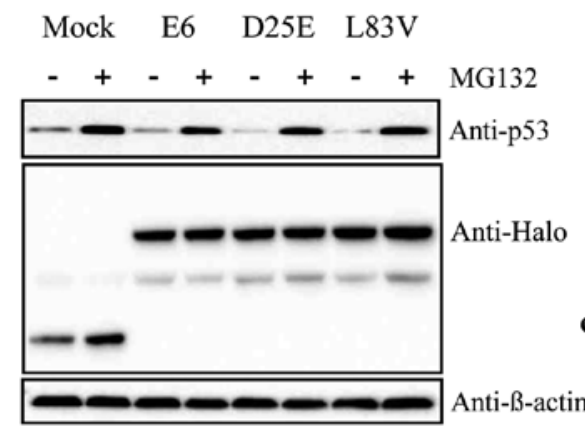

B

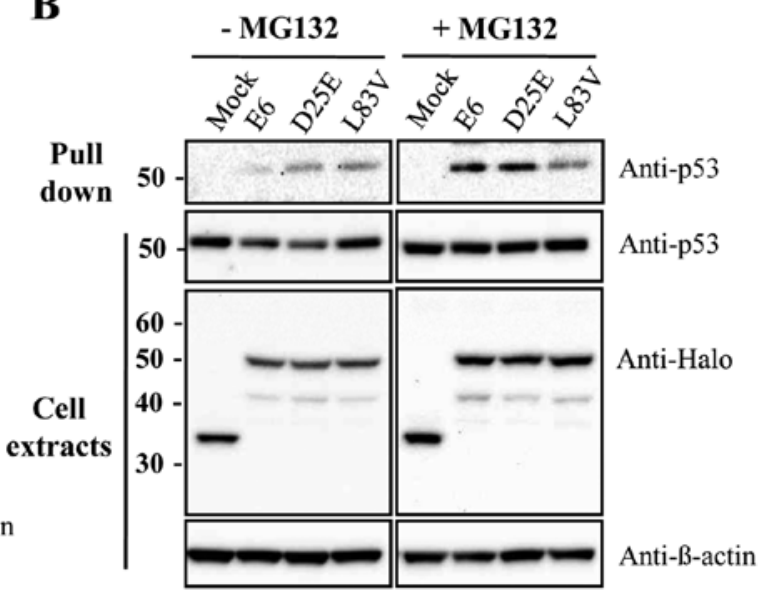

Figure 2. Overexpression of E6 prototype protein and D25E and L83V variant proteins triggers proteasome-dependent degradation of p53 via direct proteinprotein interactions. Cells transfected with the empty vector, Halo-E6, Halo-E6 D25E or Halo-E6 L83V vectors were incubated in the presence or absence of $10 \mu \mathrm{M}$ MG132 for $2 \mathrm{~h}$ before harvesting. (A) Cells were sonicated and subjected to western blot analysis using anti-p53 and anti-Halo antibodies. $\beta$-actin was used as the loading control. (B) Twenty-four hours after transfection, cell lysates were incubated with HaloLink ${ }^{\mathrm{TM}}$ resin at $4^{\circ} \mathrm{C}$. Proteins corresponding to $10 \%$ of the input and proteins retained on the resin beads were analyzed by western blot analysis with anti-p53 and anti-Halo antibodies.

HPV-negative C33A cervical carcinoma cell lines, which were transfected with the empty vector, Halo-E6, Halo-D25E and Halo-L83V constructs; prototype E6 and E6 variant proteins were detected with the anti-Halo antibody. Levels of p53 were then measured by immunoblot analysis. The expression levels of the E6 variant proteins were similar to those of the E6 prototype (Fig. 1A). As indicated in Fig. 1, all examined proteins actively promoted p53 degradation, and the level of p53 was similar in all the tested cell lines compared with the empty vector-treated samples. Degradation activities ranged between 30 and $40 \%$ of the control levels (Fig. 1B) and did not differ between the E6 prototype protein and variants. Thus, the E6 D25E and L83V variants had similar abilities to degrade p53 as the prototype protein. For further functional studies, we used HeLa cells as there were no significant differences in the rate of inactivation of p53 by E6 proteins among the cell lines studied.
E6 D25E variant interaction with p53 in vitro. The E6 viral oncoprotein produced by HR-HPV fosters ubiquitination and the proteasome-dependent degradation of p53 through proteinprotein interactions. To examine whether E6 variants, such as the E6 prototype, can reduce p53 levels via a proteasomedependent pathway, the HeLa cells were transfected with E6 variants or the E6 prototype protein as the control. At $24 \mathrm{~h}$ after transfection, the cells were then treated with the proteasome inhibitor, MG132, for $2 \mathrm{~h}$ before harvesting. As shown in Fig. 2A, the level of p53 was increased with MG132 treatment. This suggests that the reduction in p53 levels by D25E and L83V was caused by proteasome-dependent degradation. In addition, we examined whether these E6 variant proteins can regulate $\mathrm{p} 53$ protein levels by direct interaction with each other. Binding assays showed that D25E and L83V interact directly with $\mathrm{p} 53$ in the presence or absence of MG132 (Fig. 2B). The interaction was increased when the cells were treated with 
A

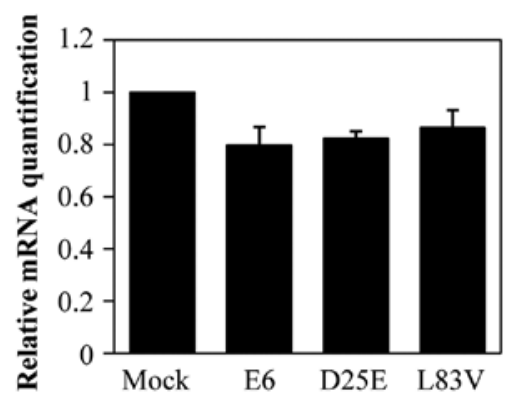

B

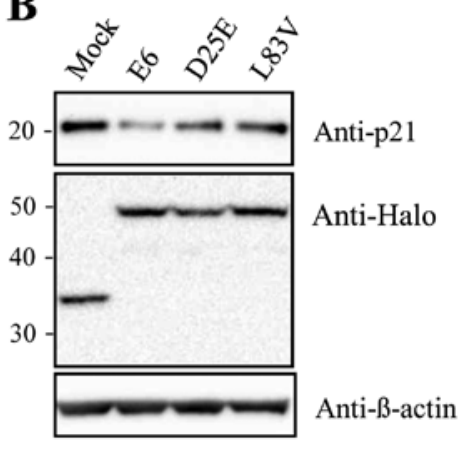

Figure 3. The levels of p21 gene expression are reduced by E6 variants. HeLa cells were co-transfected with vectors for transient expression of the E6 prototype protein and its variants. Total RNA and crude extracts were isolated $24 \mathrm{~h}$ later. (A) The total amount of p21 mRNA was measured by quantitative RT-PCR. Values were normalized to the housekeeping gene, $\beta$-actin. Numbers are the means of three independent experiments. (B) Total cell lysates were separated on $10 \%$ SDS-PAGE and analyzed by western blot analysis using a monoclonal anti-p21 antibody.

MG132, possibly due to the higher concentration of existing p53 protein. Thus, both the E6 prototype protein and its variants, D25E and L83V, affect p53 degradation by binding to p53 directly.

E6 D25E and L83V variants downregulate the induction of p21 gene expression. Abnormalities in the molecular pathways that mediate the cell cycle have been implicated in p53-regulated pathways. A well-known target of p53 is the p21 gene that causes cell cycle arrest. Therefore, we evaluated the level of p21 to determine the effect of degraded p53 in each cell line. As shown in Fig. 3, p21 mRNA and protein levels were decreased in the E6 protein-expressing cell lines. These results indicate that the downregulation of p53 by E6 proteins inhibits p21 expression. Additionally, the prototype E6 protein and its variants inhibited p21 expression in a similar manner despite different variant protein levels. These results suggest that the E6 protein can accommodate amino acid changes without significantly perturbing the activity of this protein in degrading p53 and overriding cell growth arrest via p21.

E6 D25E and L83V variants promote entry into the S phase of the cell cycle in HeLa cells. The ability of HR-HPV E6 to inhibit growth arrest and to abrogate DNA damage response induced by $\mathrm{p} 53$ and the downregulation of the p21 gene are crucial during HPV-associated carcinogenesis $(4,18)$. We examined the ability of the E6 variants to override growth arrest and determined the G1, S and G2/M phases and the $\mathrm{G} 1 / \mathrm{S}$ ratios in the E6 protein-expressing cell lines (Fig. 4A) by ModFit LT analysis using flow cytometry. HeLa cells were transfected with the E6 prototype, D25E or L83V. The expression of the E6 protein resulted in an increase in the percentage of cells at the S phase entry stage (Fig. 4B), as evidenced by an increase in the proportion of cells in the $S$ phase with an increase in the S/G1 ratio (Fig. 4C). The ratio was increased by $>20 \%$ in the E6 variant-expressing cell lines. There were no differences among the E6 protein variants, indicating that all overcame the growth arrest induced by $\mathrm{p} 21$ repression. This showed that the natural E6 variants, D25E and L83V, contribute to cell growth and proliferation through the reduction and downregulation of p53 expression.

\section{Discussion}

It has been proposed that intratypic variations of HPV types can affect their carcinogenic potential (19). The causes for the pathogenic differences between HPV-16 variants are not yet understood, although in a limited number of studies, differences in the LCR, E6, E2 and E7 sequences have been associated with altered biological functions $(19,20)$. The E6 L83V polymorphism has been reported to be associated with an increased risk of cancer progression in certain populations and has been detected at a high frequency in all European populations (21-23). This variant has been shown to enhance E6-mediated MAPK signaling and differentially regulates tumorigenesis by Notch signaling and oncogenic Ras expression (12). These and perhaps other altered functions may underlie the increased pathogenicity of L83V. However, there is no experimental evidence of whether the HPV-16 E6 D25E variant protein contributes to disease progression, as opposed to the prototype E6 protein. To our knowledge, this is the first study to determine whether the high prevalence of naturally occurring HPV-16 E6 variants in Asian and European populations differ from prototype E6 in their ability to regulate p53 expression. The D25E and L83V variants of HPV-16 E6, whose distribution in cervical precursor lesions and cancers has been determined in Asian and European populations (10,15), were compared for p53 degradation and changes in the cell cycle dynamics via p21 downregulation in E6-expressing cell lines. There were uniform patterns of activity with small differences among variants in the assays tested.

E6-mediated p53 degradation has been considered a hallmark function of oncogenic HPV types, although E6 is a highly multifunctional protein $(24,25)$. E6 proteins from HPV-16 are able to bind to $\mathrm{p} 53$ and this binding promotes the degradation of p53 via the ubiquitin pathway (26). The degradation of p53 and the induction of p53-mediated growth arrest associated with DNA damage caused by E6 are believed to contribute to the accumulation of genetic changes associated with cervical carcinogenesis (25). The variants examined in the present study contained amino acid changes in positions 25 and 83 but retained the prototype level of p53 degradation activity. Thus, similar to the prototype E6 protein, the D25E and L83V variant 

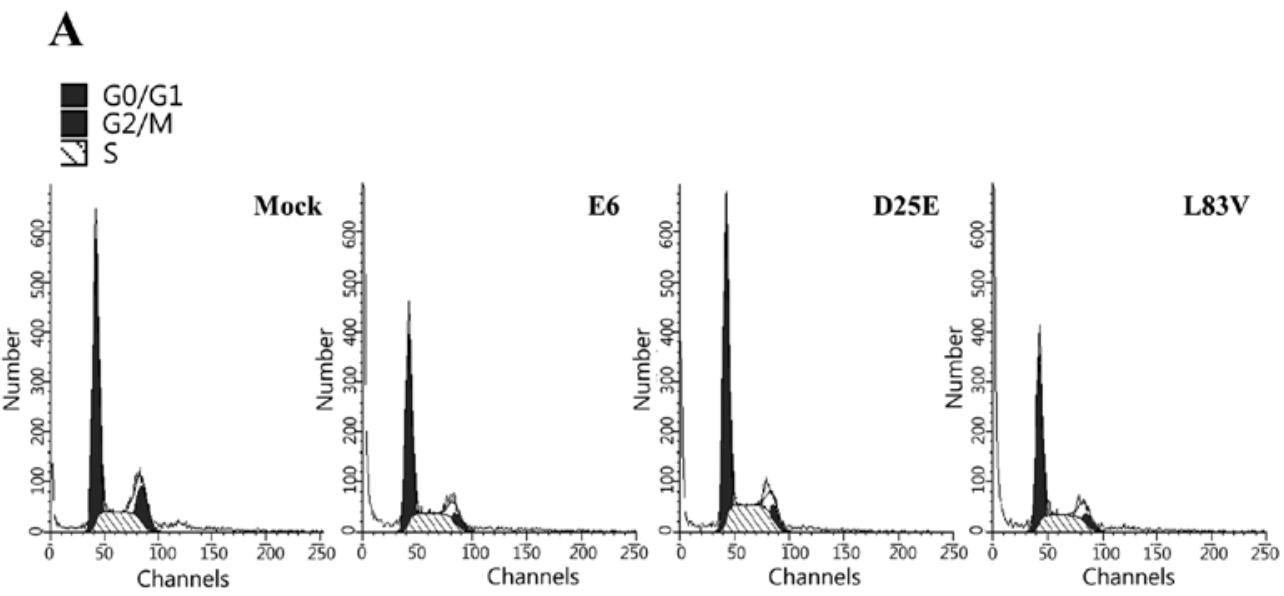

B

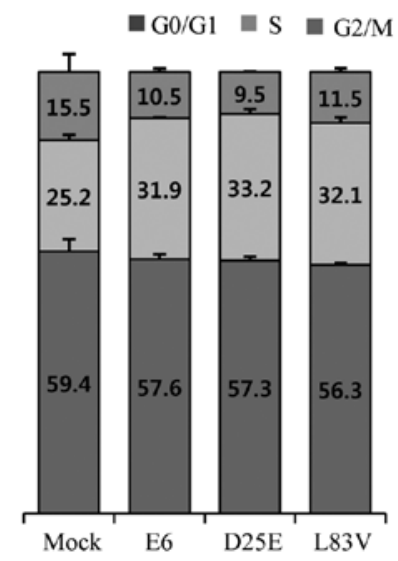

C

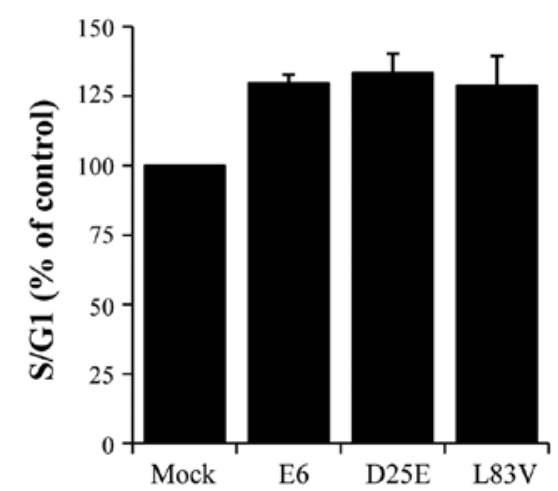

Figure 4. E6 D25E and L83V variant proteins promote cell cycle progression. The distribution of cells in G0/G1, S and G2/M phases measured by flow cytometry is demonstrated for HeLa cells transfected with prototype E6- and variant-expressing vectors or control vector (Mock). (A) Cell distributions from a representative experiment. (B) Mean percentages of cells in different stages of the cell cycle. (C) Differences in the S/G1 cell cycle ratio. Mean values of at least three independent experiments are presented as the means \pm standard deviation (SD).

proteins are also able to suppress the elevation in levels of p53 protein and to override p53-mediated growth arrest through E6-p53 interaction. The binding of the prototype E6 and the two variants with p53 occurred at similar levels. These results support the notion that the HPV-16 E6 protein can accommodate some non-conservative changes between natural variants for its interaction with p53/E6, without significant changes in its ability to target p53 for degradation. As with p53 degradation, the variants examined in this study showed similar activities in p21 expression. The p21 tumor suppressor protein is a universal inhibitor for cyclin-cyclin dependent kinase complexes and DNA replication that induces cell cycle arrest at the G1/S checkpoint. The expression of p21 is regulated transcriptionally via the $\mathrm{p} 53$ protein $(27,28)$. The repression of p21 by p53 degradation through HPV-16 E6 proteins may result in the stimulation of cell growth. To examine this possibility, we stably transfected several HeLa cell lines with constructs containing E6 proteins and measured their cell cycle profiles. As expected, the expression of the $\mathrm{p} 21$ protein was downregulated and the proportion of cells in the $S$ phase was increased in each E6-expressing cell line. However, the results were similar between the E6 prototype protein and its variants. These results are consistent with those from a previous study (28), indicating that HPV-16 E6 variant proteins repress the transcription of p21 by the degradation of the p53 protein via protein-protein interaction(s) and reduce the level of p21 protein through transcriptional repression among different E6 protein-expressing cell lines.

The similarities in the modulation of DNA damage responses between the E6 prototype and its variants, L83V and D25E, both in terms of suppression of p53 accumulation and overcoming growth arrest through p21 downregulation, strongly suggest that these functions of E6 cannot be compromised to initiate carcinogenesis.

In conclusion, using functional assays, we show that naturally occurring amino acid variations in the E6 protein affect pathogenesis by $\mathrm{p} 53$-associated proteins in human cervical cancer cell lines. The ability of the proteins to induce p53 degradation and modulate cell cycle profiles via $\mathrm{p} 21$ repression in various HPV-16 E6 variant protein-expressing HeLa cells was similar to the prototype E6 protein. Thus, the inactivation of p53 by E6 variants may contribute to immortalization by preventing the cells from arresting in response to genomic instability, in a similar manner to the E6 prototype protein. To the best of our knowledge, this is the first study to examine whether the high prevalence of naturally occurring HPV-16 E6 variants in Asian and European populations differ from the prototype E6 protein in their ability to regulate p53 expression. 
The data presented in this study may open the way for future mechanistic studies. Naturally occurring variants may display biological differences other than those described in this study, which could contribute to their pathogenicity. Further structural and biochemical analyses with E6 variants are warranted to improve our understanding of their biological functions and epidemiology, and of how they modulate the progression of carcinogenesis.

\section{Acknowledgements}

This study was supported by a grant from the health Promotion against HIV/AIDS and STD (4800-4842-302) and from the Pathogenic Proteome Management Program (4800-4847-300) of the National Institute of Health, Ministry of Health and Welfare, Republic of Korea.

\section{References}

1. Bosch FX, Manos MM, Muñoz N, et al: Prevalence of human papillomavirus DNA in cervical cancer: a worldwide perspective. International biological study on cervical cancer (IBSCC) study group. J Natl Cancer Inst 87: 796-802, 1995.

2. Liu Y, Chen JJ, Gao Q, et al: Multiple functions of human papillomavirus type 16 E6 contribute to the immortalization of mammary epithelial cells. J Virol 73: 7297-7307, 1999.

3. Lehoux M, D'Abramo CM and Archambault J: Molecular mechanisms of human papillomavirus-induced carcinogenesis. Public Health Genomics 12: 268-280, 2009.

4. Moody CA and Laimins LA: Human papillomavirus oncoproteins: pathways to transformation. Nat Rev Cancer 10: 550-560, 2010.

5. Huibregtse JM, Scheffner M and Howley PM: A cellular protein mediates association of $\mathrm{p} 53$ with the E6 oncoprotein of human papillomavirus types 16 or 18. EMBO J 10: 4129-4135, 1991 .

6. Huertas-Salgado A, Martín-Gámez DC, Moreno P, et al: E6 molecular variants of human papillomavirus (HPV) type 16: an updated and unified criterion for clustering and nomenclature Virology 410: 201-215, 2011.

7. Ellis JR, Etherington I, Galloway D, et al: Antibody responses to HPV16 virus-like particles in women with cervical intraepithelial neoplasia infected with a variant HPV16. Lancet 34: 1069-1070, 1997.

8. Zehbe J, Mytilineos I, Wikstrom R, et al: Association between human papillomavirus 16 E6 variants and human leukocyte antigen class I polymorphism in cervical cancer of Swedish women. Hum Immunol 64: 538-542, 2003.

9. Andersson S, Alemi M, Rylander E, et al: Uneven distribution of HPV 16 E6 prototype and variant (L83V) oncoprotein in cervical neoplastic lesions. Br J Cancer 83: 307-310, 2000.

10. Zehbe I, Wilander E, Delius H, et al: Human papillomavirus 16 E6 variants are more prevalent in invasive cervical carcinoma than the prototype. Cancer Res 58: 829-833, 1998.
11. Stoppler MC, Ching K, Stoppler H, et al: Natural variants of the human papillomavirus type 16 E6 protein differ in their abilities to alter keratinocyte differentiation and to induce p53 degradation. J Virol 70: 6987-6993, 1996.

12. Chakrabarti O, Veeraraghavalu K, Tergaonkar V, et al: Human papillomavirus type 16 E6 amino acid 83 variants enhance E6-mediated MAPK signaling and differentially regulate tumorigenesis by notch signaling and oncogenic Ras. J Virol 78: 5934-5945, 2004

13. Cai HB, Chen CC and Ding XH: Human papillomavirus type 16 E6 gene variations in Chinese population. Eur J Surg Oncol 36: 160-163, 2010.

14. Matsumoto K, Yoshikawa H, Nakagawa S, et al: Enhanced oncogenicity of human papillomvairus type 16 (HPV16) variants in Japanese population. Cancer Lett 56: 159-165, 2000.

15. Kang S, Jeon YT, Kim JW, et al: Polymorphism in the E6 gene of human papillomavirus type 16 in the cervical tissues of Korean women. Int J Gynecol Cancer 15: 107-112, 2005.

16. Jang M, Rhee JE, Jang DH and Kim SS: Gene expression profiles are altered in human papillomavirus-16 E6 D25E-expressing cell lines. Virol J 8: 453, 2011.

17. Thomas M, Pim D and Banks L: The role of the E6-p53 interaction in the molecular pathogenesis of HPV. Oncogene 18: 7690-7700, 1999 .

18. Kessis TD, Slebos RJ, Nelson WG, et al: Human papillomavirus 16 E6 expression disrupts the p53-mediated cellular response to DNA damage. Proc Natl Acad Sci USA 90: 3988-3992, 1993.

19. Bernard HU, Calleja-Macias IE and Dunn ST: Genome variation of human papillomavirus types: phylogenetic and medical implications. Int J Cancer 118: 1071-1076, 2006.

20. Hildesheim A and Wang SS: Host and viral genetics and risk of cervical cancer: a review. Virus Res 89: 229-240, 2002.

21. Zehbe I, Voglino G, Delius H, et al: Risk of cervical cancer and geographical variations of human papillomavirus 16 E6 polymorphisms. Lancet 352: 1441-1442, 1998.

22. Kämmer C, Tommasino M, Syrjänen S, et al: Variants of the long control region and the E6 oncogene in European human papillomavirus type 16 isolates: implications for cervical disease. Br J Cancer 86: 269-273, 2002.

23. Grodzki M, Besson G, Clavel C, et al: Increased risk for cervical disease progression of French women infected with the human papillomavirus type 16 E6-350G variant. Cancer Epidemiol Biomarkers Prev 15: 820-822, 2006.

24. McLaughlin-Drubin ME and Munger K: Oncogenic activities of human papillomaviruses. Virus Res 143: 195-208, 2009.

25. Howie HL, Katzenellenbogen RA and Galloway DA: Papillomavirus E6 proteins. Virology 384: 324-334, 2009.

26. Scheffner M, Werness BA, Huibregtse JM, Levine AJ and Howley PM: The E6 oncoprotein encoded by human papillomavirus types 16 and 18 promotes the degradation of p53. Cell 63: 1129-1136, 1990.

27. Rowland BD and Peeper DS: KLF4, p21 and context-dependent opposing forces in cancer. Nat Rev Cancer 6: 11-23, 2006.

28. Munger K and Howley PM: Human papillomavirus immortalization and transformation functions. Virus Res 89: 213-228, 2002. 